\title{
Observing Quanta on a Cosmic Scale
}

\author{
Craig J. Hogan \\ Astronomy and Physics Departments, University of Washington, Seattle, Washington 98195-1580
}

\begin{abstract}
Our entire galaxy, like all others, originated as a fairly smooth patch of binding energy, which in turn originated as a single quantum perturbation of the inflaton field on a subatomic scale during inflation. The best preserved relic of these perturbations is the anisotropy of the microwave background radiation, which on the largest scales preserves a faithful image of the primordial quantum fields. It is possible that close study of these perturbations might reveal signs of discreteness caused by spacetime quantization.
\end{abstract}

\section{COSMIC BACKGROUND ANISOTROPY: IMAGES OF INFLATON QUANTA ON THE SKY}

At the beginning of the first session of the Humboldt Foundation's symposium "Werner Heisenberg: 100 years Works and Impact", an eminent particle theorist chose to begin the conference with a talk not on the grand tradition of Heisenberg quantum mechanics we had gathered to celebrate, but on the much less elegant topic of astrophysical cosmology. This choice made perfect sense: at this moment in the development of science, the most fundamental advances in understanding the innermost world of small things are coming from studies of big things: The newest physics is coming from astrophysics.

The last few years have seen spectacular discoveries of this kind. Astrophysical experiments have determined that neutrinos have mass, demonstrating the first new extension of Standard Model phenomena in many years. The mass-energy of the universe appears to be dominated by a new form of "Dark Energy" with a sufficiently large repulsive gravitational effect to accelerate the cosmic expansion of the universe, as detected in measurements of distant supernovae, and which defies all attempts at a deep theoretical understanding. Another great advance, the detailed measurement of the pattern of primordial anistropy in the cosmic microwave background, has now started to nail down basic parameters of the universe with some precision. We now know for example that the large scale geometry of the universe is nearly flat, or to put it another way, the entire cosmic hypersphere appears to be at least ten times larger in linear scale than the piece accessible to our telescopes. Better data soon to come will probably drive that lower limit up to a factor of a hundred. Real data are confirming the expectation, from inflation theory, that the universe is much larger than what we can ever see.

From the point of view of Heisenberg, perhaps most amazing newly discovered phenomena are the perturbations that create the anisotropy. Hot and cold patches in the cosmic background radiation correspond to small fractional perturbations in gravitational potential, on a vast scale, with coherent patches stretching across the entire visible universe. The perturbations are also ultimately responsible for causing all of the structure in the universe, the superclusters of galaxies, and the galaxies, stars and planets within them. They are most remarkable however because they are a quantum phenomenon: the pattern of hot and cold patches scientists map on the sky today is a faithful image of the configuration of quantum field fluctuations that occurred in a tiny patch of space, far smaller than an atom, long ago during cosmic inflation. Roughly speaking, each hot and cold patch originated as a single quantum of the inflaton field, which was subsequently enormously magnified by the cosmic expansion acting as a coherent, nearly noise-free amplifier.

This audacious application of quantum mechanics is generally accepted as the correct explanation of the origin of these perturbations because a well-controlled model [1] 8] makes definite predictions that agree well with the data [9 14. The basic framework of the theory consists of free relativistic quantum fields in a classical curved spacetime: that is, Einstein's classical theory of gravity is used to compute the spacetime background on which the quantum fields propagate. The fields affect the spacetime through their energy-momentum tensor, as described by the Einstein field equations, but the spacetime itself is not quantized. The fields approximate a collection of quantized harmonic oscillators in an expanding background, with perturbations due to the zero-point field amplitudes in their ground state. Each mode expands with the background universe, and at a certain point its fluctuations are "frozen in" as its oscillation rate becomes smaller than the expansion rate, a process that can most accurately be descibed as a phase wrapping or state squeezing $[15,20]$. The fluctuation becomes a perturbation in the spacetime metric [2, which continues to expand by a huge factor as the universe exponentially increases in size during inflation. The final superposition of all the modes creates metric perturbations described as a continuous field with random Gaussian statistics, and a nearly (though not exactly) scale-free spectrum. These predictions are confirmed by the current data.

Thus the structure of the universe on the largest scales is directly connected to quantum processes on the smallest scales. The statistics of the fluctuations, such as the amplitude and slope of their spectrum, are determined by certain 
combinations of parameters of the field Lagrangian. The data on anisotropy provide by far the most precise data we have on the structure of fundamental forces on such small scales (albeit, the information is limited in scope to a few special combinations of parameters).

Indeed, the theory extrapolates the basic theoretical framework tens of orders of magnitude from any other experimental data, which leads to some healthy scepticism about whether we ought to believe it at all. In some situations (in particular, if inflation occurs close enough to the Planck scale to produce detectable and separable tensor perturbations due to graviton fluctuations, in addition to the scalar modes that lead to galaxies), we may be able to implement a more detailed test, for example, a comparison of the relative amplitude of scalar and tensor mode perturbations with the slope of the spectrum [21,22]. However, for plenty of choices of parameters the field theory framework predicts only unobservable departures from scale-invariant, random Gaussian noise.

\section{HOLOGRAPHIC INFORMATION BOUND AND QUANTUM DISCRETENESS OF ANISOTROPY}

It has always been acknowledged that standard inflation theory is only itself an approximation, because it does not include quantization of spacetime itself - and for good reason, since there is not yet a widely accepted theory of quantum gravity. On the other hand, there are now some definite quantitative results in quantum gravity: the dimension of the Hilbert space is known or bounded, giving bounds on the total entropy and information accessible to all configurations of all fields, including the quantum degrees of freedom of the spacetime itself. Instead of having an arbitrary zero point, entropy can now be defined in absolute terms. In other words, there are absolute limits on how many different things can happen within the confines of any given region.

This in turn imposes bounds on everything during inflation, including the behavior of free quantum fields. Their modes are not truly and fundamentally independent as assumed in the standard picture, nor do they have an infinite Hilbert space. This is a radical departure from the foundations of field theory, but it is required if we wish to include spacetime as a quantum object in the fundamental theory, in particular one that obeys unitary evolution without fundamental loss of information.

Consider for example the thought experiment of a black hole that forms, then evaporates via Hawking radiation $22328]$. If the whole process is unitary 29,32 , then the states of radiated particles depend in detail on how the hole was formed. Indeed, running things backwards in time, carefully assembled" incoming particles would form a small black hole that grows and then disassembles by throwing out any particular macroscopic objects - TV sets, whateverthat went into the hole. This is only possible if the spacetime metric encodes at a fundamental level the information equivalent to the radiated entropy. The entropy is known from thermodynamic arguments to be one quarter of the area of the event horizon in Planck units. Since a black hole is the highest entropy state attainable by any amount of mass/energy, one is led to the "Holographic Principle" 33 35]: for any physical system, the total entropy $S$ within any surface is bounded by one quarter of the area $A$ of the surface in Planck units (adopting $\hbar=c=G=1$ ). This is an "absolute" entropy; the dimension $N$ of the Hilbert space is given by $e^{S}$, and the total number of distinguishable quantum states available to the system is given by a binary number with $n=S / \ln 2=A / 4 \ln 2$ digits 36 .

A holographic bound can also be derived for the universe as a whole [37 42]. Because the cosmological solutions do not have the asymptotically flat infinity of the black hole solutions for defining particle states, the bound has a somewhat different operational meaning, referring to an "observable entropy". The cosmological version [43,44] of the holographic principle is: the observable entropy of any universe cannot exceed $S_{\max }=3 \pi / \Lambda$, where $\Lambda$ is the cosmological constant in Planck units. In a de Sitter universe, as in a black hole, this corresponds to one quarter of the area of the event horizon in Planck units, but the bound is conjectured to hold for any spacetime, even FriedmannRobertson-Walker universes with matter as well as $\Lambda$. The inflationary part of our spacetime closely resembles a piece of a de Sitter universe, so there is a bound on the observable entropy of all quantum fields during inflation: $S_{\max }=\pi / H^{2}$, where $H$ is the expansion rate during inflation. That means that all the modes of all the fields have the same size Hilbert space as a system of $n=\pi / H^{2} \ln 2$ binary spins.

The value of $H$ during inflation is not known, but the field theory predicts that graviton fluctuations are produced with amplitude $\approx H$, leading to tensor perturbations of the metric and anisotropy with amplitude $\delta T / T \approx H$; since the observations with $\delta T / T \approx 10^{-5}$ are dominated by scalar perturbations, we currently have a bound around

$H \leq 10^{-5}$. (This bound will improve with the advent of experiments with better sensitivity to polarization that

\footnotetext{
${ }^{1}$ To do this right with the known CP-violating, CPT-invariant fields, one would have to take the final states and parity reverse them before running them backwards in time.
} 
can separate tensor and scalar components [45). In round numbers then, the universe during inflation has a Hilbert space equivalent to at least $10^{10}$ spins. That is unquestionably a large number, but then again it is much smaller than the infinite Hilbert space of the field theory description. It is also much smaller than the Hilbert space of other astrophysical systems, such as stellar-mass black holes.

The most interesting question is, can we detect any observable effect of the finite Hilbert space on the fluctuations? We derive some hope from the fact the the microwave background experiments have such a high precision, and that the effects of various complicated astrophysical foregrounds have been successfully removed to reveal the simple, cleanly modeled perturbations in the cosmic last scattering surface.

To get one very rough estimate of the possible amplitude of the effect, consider the following crude model. Pay attention only to one set of modes, those five independent components that contribute to the observed quadropole moment of the anisotropy. These perturbations are created by (and are amplified images of) quantum fluctuations around the time that the current Hubble length passed through the inflationary event horizon; the amplitude of the observed temperature perturbation traces in detail a quantity which is proportional to the amplitude of the quantum field. Imagine that these modes contain all of the information allowed by the cosmological holographic bound, and further that they are literally composed of $n$ pixels on the sky, each of which represents a binary spin. For the maximal value $H \approx 10^{-5}$ (the maximum allowed by the tensor-mode limits), there would be of order $10^{10}$ "blackand-white" pixels, corresponding to a pixel scale of only a few arc seconds.2 This level of discreteness would not be observable in practical terms, for two reasons: the last scattering surface is much thicker than this (which smears out the contribution of many pixels due to optical transfer and acoustic effects at a redshift of about 1000), and there are many other modes on a smaller scale superimposed.

On the other hand, at least one important assumption in this estimate is likely to be wrong by orders of magnitude: the modes on the horizon scale probably carry only a small fraction of the holographic information bound. At any given time, most of the information is in much smaller wavelength modes. A toy model of spacetime discreteness 46 ] suggests that the Hilbert space of the horizon-scale quantum perturbations is equivalent to at most only about $10^{5}$ binary spins. (This number is determined not the tensor-mode limit on $H$, but by the inverse of the observed scalar perturbation amplitude). This in principle may produce observable discreteness, since the all-sky cosmic background anisotropy includes about $10^{4}$ independent samplings of inflationary fields (determined by the angular size of the horizon at last scattering).

Now the idea that there are literally binary pixels is also silly, and was used here only for illustrative convenience. In fact we have no clear idea of the actual character of the holographic eigenstates projected onto the inflaton perturbations; we have only the counting argument to guide us. In [46], a toy model assumed that $H$ and $\delta T$ come in discrete levels, but this again was only for calculational convenience. In the absence of a more concrete theory of the nature of the holographic modes, it makes sense to consider a variety of tests on the data to seek departures from the field-theory prediction of a continuous, random Gaussian field. The most straightforward check will be to test whether the amplitudes of the harmonics $A_{\ell m}$ come in discrete values rather than being selected from a random continuous Gaussian distribution. Although this test could already be performed fairly reliably using the all-sky COBE satellite data [47,48], it will become much more powerful using the all-sky data from the MAP (and later PLANCK) satellites, which will have much finer angular resolution capable of cleanly resolving modes far below the horizon scale at recombination (a level already reached in ground- and balloon-based experiments with limited sky coverage). The high quality of this data will let us search for true quantum-gravity effects.

\section{ACKNOWLEDGMENTS}

I am grateful for useful conversations with J. Bardeen, R. Bousso, and R. Brandenberger. This work was supported by NSF grant AST-0098557 at the University of Washington.

\footnotetext{
${ }^{2}$ This level of graininess in the everyday world would be just below the level of detectability with the naked eye.
} 
[1] A. A. Starobinsky, JETP Lett. 30, 682 (1979) [Pisma Zh. Eksp. Teor. Fiz. 30, 719 (1979)].

[2] J. M. Bardeen, Phys Rev D 22, 1882 (1980)

[3] S. W. Hawking, Phys. Lett. B 115, 295 (1982).

[4] A. H. Guth \& S. Y. Pi, Phys. Rev. Lett. 49, 1110 (1982).

[5] J. M. Bardeen, P. J. Steinhardt \& M. S. Turner, Phys. Rev. D 28, 679 (1983).

[6] A. A. Starobinsky, Phys. Lett. B 117, 175 (1982).

[7] J. J. Halliwell \& S. W. Hawking, Phys. Rev. D 31, 1777 (1985).

[8] L. P. Grishchuk, Class. Quant. Grav. 10, 2449 (1993)

[9] G.F.Smoot et al.,Astrophys.J. 396, L1(1992)

[10] P. de Bernardis et al., Nature 404 (2000) 955-959

[11] C. Pryke et al., Ap. J. in press, astro-ph/0104490 (2001)

[12] N. W. Halverson et al., Angular astro-ph/0104489 (2001)

[13] S. Hanany et al., Astrophys.J. 545, L5 (2000)

[14] J. R. Bond \& R. G. Crittenden, "CMB Analysis", in Proc. NATO ASI "Structure Formation in the Universe", eds. R.G. Crittenden \& N.G. Turok, astro-ph/0108204, (Kluwer, 2001)

[15] L. P. Grishchuk \& Y. V. Sidorov, Class. Quant. Grav. 6, L161 (1989).

[16] L. P. Grishchuk \& Y. V. Sidorov, Phys. Rev. D 42, 3413 (1990).

[17] A. Albrecht, P. Ferreira, M. Joyce \& T. Prokopec, Phys. Rev. D 50, 4807 (1994)

[18] J. Lesgourgues, D. Polarski \& A. A. Starobinsky, Nucl. Phys. B 497, 479 (1997)

[19] D. Polarski \& A. A. Starobinsky, Class. Quant. Grav. 13, 377 (1996)

[20] C. Kiefer, D. Polarski \& A.A. Starobinsky, Int.J.Mod.Phys. D7, 455-462 (1998)

[21] V. F. Mukhanov, H. A. Feldman \& R. H. Brandenberger, Phys. Rept. 215, 203 (1992)

[22] D. H. Lyth \& A. Riotto, Phys.Rept. 314, 1-146 (1999)

[23] J. D. Bekenstein, Lett. Nuovo Cim. 4, 737 (1972)

[24] J. D. Bekenstein, Phys. Rev. D 7, 2333 (1973)

[25] J. D. Bekenstein, Phys. Rev. D 9, 3292 (1974)

[26] S. W. Hawking, Commun. Math. Phys. 43, 199 (1975)

[27] S. W. Hawking, Phys. Rev. D 14, 2460 (1976)

[28] J. D. Bekenstein, Phys. Rev. D 23, 287 (1981)

[29] T. Banks, L. Susskind \& M. E. Peskin, Nucl. Phys. B 244, 125 (1984)

[30] G. 't Hooft, Nucl. Phys. B 256, 727 (1985)

[31] L. Susskind, L. Thorlacius \& J. Uglum, Phys. Rev. D 48, 3743 (1993)

[32] C.R. Stephens, G. 't Hooft, \& B.F. Whiting, Class. Quant. Grav. 11, 621-648 (1994)

[33] G. 't Hooft, "Dimensional Reduction in Quantum Gravity", essay in honor of Abdus Salam, gr-qc/9310026 (1993)

[34] L. Susskind, J.Math.Phys. 36, 6377 (1995)

[35] D. Bigatti and L. Susskind, "TASI lectures on the holographic principle," arXiv:hep-th/0002044 (1999)

[36] J. D. Bekenstein "Quantum Information and Quantum Black Holes", Lectures delivered at NATO Advanced Study Institute: International School of Cosmology and Gravitation: 17th Course of the International School of Cosmology and Gravitation, Erice, May 1-11, 2001, to appear in Advances in the Interplay between Quantum and Gravity Physics, ed. V. de Sabbata (Kluwer, Dordrecht 2002)

[37] L. Susskind \& E. Witten "The Holographic Bound in Anti-de Sitter Space", hep-th/9805114 (1998)

[38] W. Fischler \& L. Susskind "Holography and Cosmology", hep-th/9806039 (1998)

[39] R. Bousso, JHEP 9907, 004 (1999)

[40] R. Bousso, JHEP 9906, 028 (1999)

[41] R. Bousso, Class. Quant. Grav. 17, 997 (2000)

[42] R. Bousso, Phys.Rev. D60, 063503 (1999)

[43] R. Bousso, "Positive Vacuum Energy and the N-bound", JHEP in press, hep-th/0010252 (2001)

[44] E. E. Flanagan, D. Marolf, R. M. Wald, Phys.Rev. D62, 084035 (2000)

[45] M. Kamionkowski \& A. Kosowski, Ann.Rev.Nucl.Part.Sci. 49, 77 (1999)

[46] C. Hogan, "Holographic Discreteness of Inflationary Perturbations", Phys. Rev. D. (submitted)

[47] C. L. Bennett et al., Astrophys. J. 464, L1 (1996)

[48] K. M. Gorski et al., Astrophys. J. 464, L11 (1996) 